\title{
Non-destructive Tracing of a Product Life Cycle Through Geometry Extraction from Radiographs
}

\author{
J. Hefele, R. D. Bolton \\ Los Alamos National Laboratory, Los Alamos, NM, USA \\ Em: jill@lanl.gov and rbolton@lanl.gov
}

Keywords Photogrammetry, Geometry Rconstruction, Non-destructive Testing

\begin{abstract}
Tracing the internal changes seen by a product assembly during its useful life cycle without disassembling or destructively testing the product provides many challenges. When an assembly is disassembled for evaluation, the effect of component interaction is lost, particularly if operations such as press-fitting have been used in the assembly process. It is undesirable to destructively evaluate a product when it is still in its useful life cycle. Los Alamos National Laboratory is developing a process in which radiographic information is used to characterise an entire assembly for life cycle evaluation and the geometric information is extracted for input into a Computer Aided Engineering (CAE) software package. Digitized radiographs have been reconstructed into solid models using FotoG ${ }^{\mathrm{TM}}$ geometry extraction techniques and ProENGINEER ${ }^{\mathrm{TM}}$ solid modelling capabilities. Executed simultaneously, FotoG ${ }^{\mathrm{TM}}$ translates the extracted geometry into a format useable by the ProENGINEER ${ }^{\mathrm{TM}}$ software. Once the solid model is constructed, it is used for creation of finite element analysis input, virtual environment model creation, and tooling design. The creation of the finite element model allows us to forestall future problems using predictive analyses. Virtual environment creation provides us with the capability of creating a learning environment for technicians performing maintenance or disassembly of problem units. And tooling design provides us the capability to safely transport severely damaged assemblies in the event of an accident scenario.
\end{abstract}

\section{INTRODUCTION}

As a product matures through its useful life cycle, characterisation of the product's current state becomes increasingly essential in order to forestall function and servicing problems. "As-Built" and "As-Is" characterisation methodologies have been developed recently [1] [2] [3] using inspection 
data as the raw input in modelling a product. This type of characterisation works well for portrayal of a component's current state. However, once components are assembled into a final product, the geometry can easily be modified due to the processes it has been subjected to during assembly or because of interactions with other components owing to use.

Characterisation of a product in its assembled state provides it's own challenges. Capturing the "As-Is" state of components in the assembled configuration requires non-destructive methods of evaluation. Radiography and Computed Tomography [4] can provide the raw data necessary for this type of characterisation. We have concentrated our initial development efforts on the use of radiographic data because much of our historical data exists in this state. Changes can occur to component geometry as a product moves through its useful life cycle and these variations from the "As-Built" state can also be captured using non-destructive evaluation techniques, thus allowing life-cycle data points to be collected as an assembly ages.

This paper will examine the data collection and manipulation processes for characterisation of a product as it moves through its useful life cycle using photogrammetric, radiographic, and computer-aided engineering modelling techniques. We will also inspect issues related to data collection methods employed to obtain the best possible data for use in product model reconstruction.

\section{DATA COLLECTION PROCESS}

The data collection process for model reconstruction of a product consists of three steps; initial survey of data collection environment, documentation of environment through digital photographs, and radiography of the product being examined.

\subsection{Initial Survey}

The initial survey of the data collection environment establishes a network of control points to be used as reference between the digital photographs taken in the next step and the real world. A theodolite is used to measure the location and orientation of the control points placed in the data collection environment prior to photography. A minimum of four control points is required. Stick-on targets are also set and located using the theodolite or by measuring the distance between control points/targets and establishing an arbitrary but correctly scaled co-ordinate system during this phase. The targets are used as references in the documentary photographs. 
Theodolite measurements can be made at this point to locate the radiographic film holder and the $\mathrm{x}$-ray source.

\subsection{Environment Documentation Using Digital Photographs}

Once the control points and targets have been established, the environment can be documented using digital photography. The assembly to be evaluated must be visible in at least two of the photographs taken with different perspectives. It is essential that the camera/lens combination used to take the photographs in this step have been calibrated using test photographs of a specially designed calibration field. Analysis of the digital photographs is benefited through inclusion of a fair amount of overlap in the photographs taken. Section 4 discusses this in more detail.

\subsection{Radiography}

Radiographic data provides the true basis for reconstruction of the assembly of interest. The same principles apply to radiographic documentation for assembly reconstruction as do to environment documentation for site reconstruction; i.e. more overlap in radiographic images, the better.

\section{DATA MANIPULATION}

Once the raw data is collected in the form of survey data, digital photographs, and digital (or digitised) radiographs, the reconstruction can begin. Analysis begins with identification of the camera/lens combination used to collect the digital photographs. This provides the FotoG ${ }^{\mathrm{TM}}$ software with the necessary information for resection of the images. Images are mathematically resected as a set with ties to control points. The analytical process is well-documented on Vexcel's web site [5]. Radiographic images can be over-laid into the digital photograph site reconstruction data or can be reconstructed as a separate data set if survey data has been collected documenting the location of the $x$-ray source and radiographic film frame.

\section{DATA COLLECTION ISSUES}

Data collection techniques effect the results obtained during the reconstruction process. The type of targets used, for example, determine the ease with which points are identified in the digital photographs while processing the data. Additionally, the precision of the transit system used, completeness of the survey techniques, choice of camera locations, number 
of control points identified, number of images used in data processing, and type of image geometry used all effect the final results obtained in the data set analysis..

\subsection{Targets}

Figure 1 illustrates examples of types of targets used. Of the three types of targets shown, we have found the easiest to work with in the photogrammetry environment is on the extreme right.

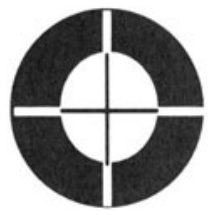

001
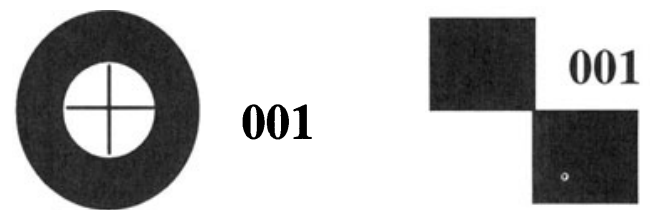

Figure 1 Examples of Target Geometry.

\subsection{Transit System Measurement Precision}

The precision inherent in the theodolite used to take measurements during the initial survey stage will effect the precision in measurements taken digitally in the reconstructed data set. It is, therefore, essential to chose a transit system with as high a precision as possible. Figure 2 compares the same measurements taken in two different data sets by the same theodolite. The error, $2-3 \mathrm{~mm}$, is within the stated precision of stated by the manufacturer.

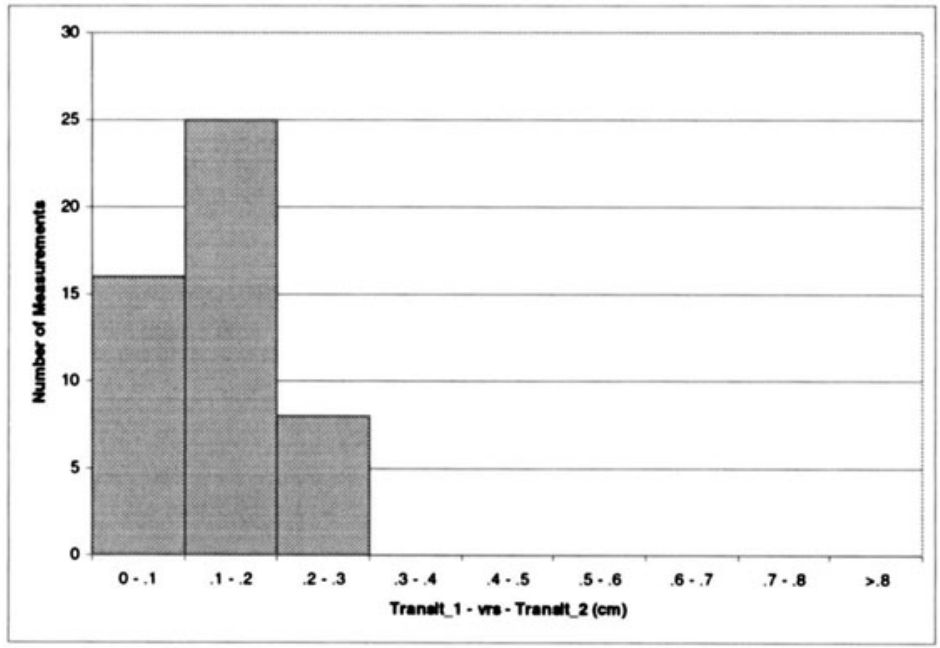

Figure 2 Comparison of Transit Measurements. 


\subsection{Photogrammetry Measurement Precision}

The precision of the photogrammetric software reconstruction is also an important issue when choosing tools to be used for reconstruction efforts. Figure 3 compares the differences observed in measurements reconstructed from digital images using the FotoG ${ }^{\mathrm{TM}}$ software with the same measurements made with the theodolite.

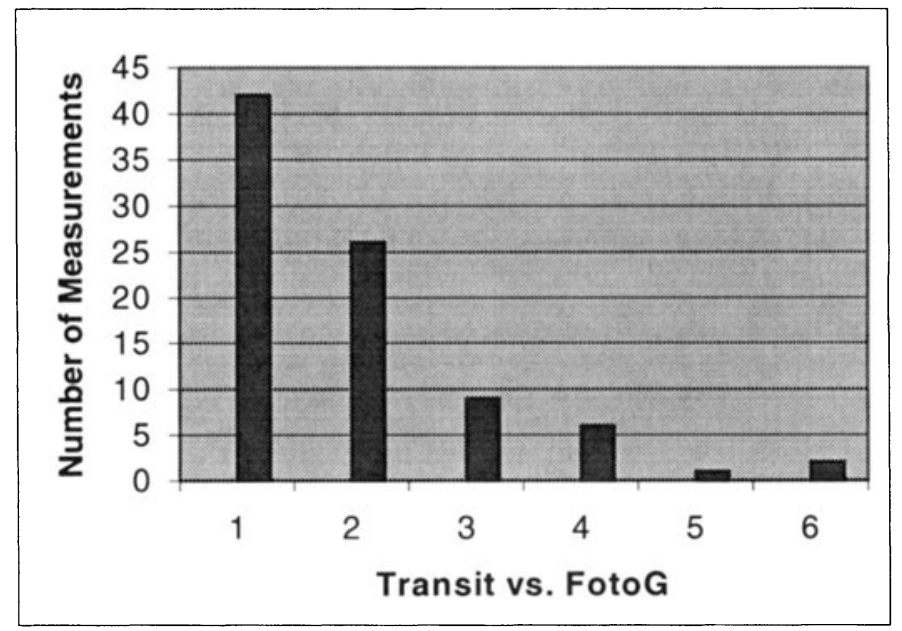

Figure 3 Comparison of Transit Measurements with FotoG Software.

\subsection{Number of Control Points vs. Precision}

The system of control points established in the initial survey during a reconstruction effort establishes the reference between the digitally documented environment and the "As-Is" condition that exists in the real world. The number of control points used has an effect on the precision attained during reconstruction. A minimum of four control points is required. Figure 4 shows the effect of increasing the number of control points used during a reconstruction from four to eight to sixteen to thirtytwo. In general, the more control points used, the better. However, there is a break even point where increasing the number of control points has only a small benefit to increasing precision.

\subsection{Number of Images vs. Precision}

The data collection process can be the most time-consuming element of a reconstruction effort. Documentation of the site and assembly environments in a complete manner is extremely important to the resulting analysis. The number of images, however, is not nearly as important as the quality of the images taken. A few images with good overlap, say greater 
than $60 \%$ coverage, and strong geometry, approximately $90 \%$ convergence angles, will give the best results in a reconstruction.
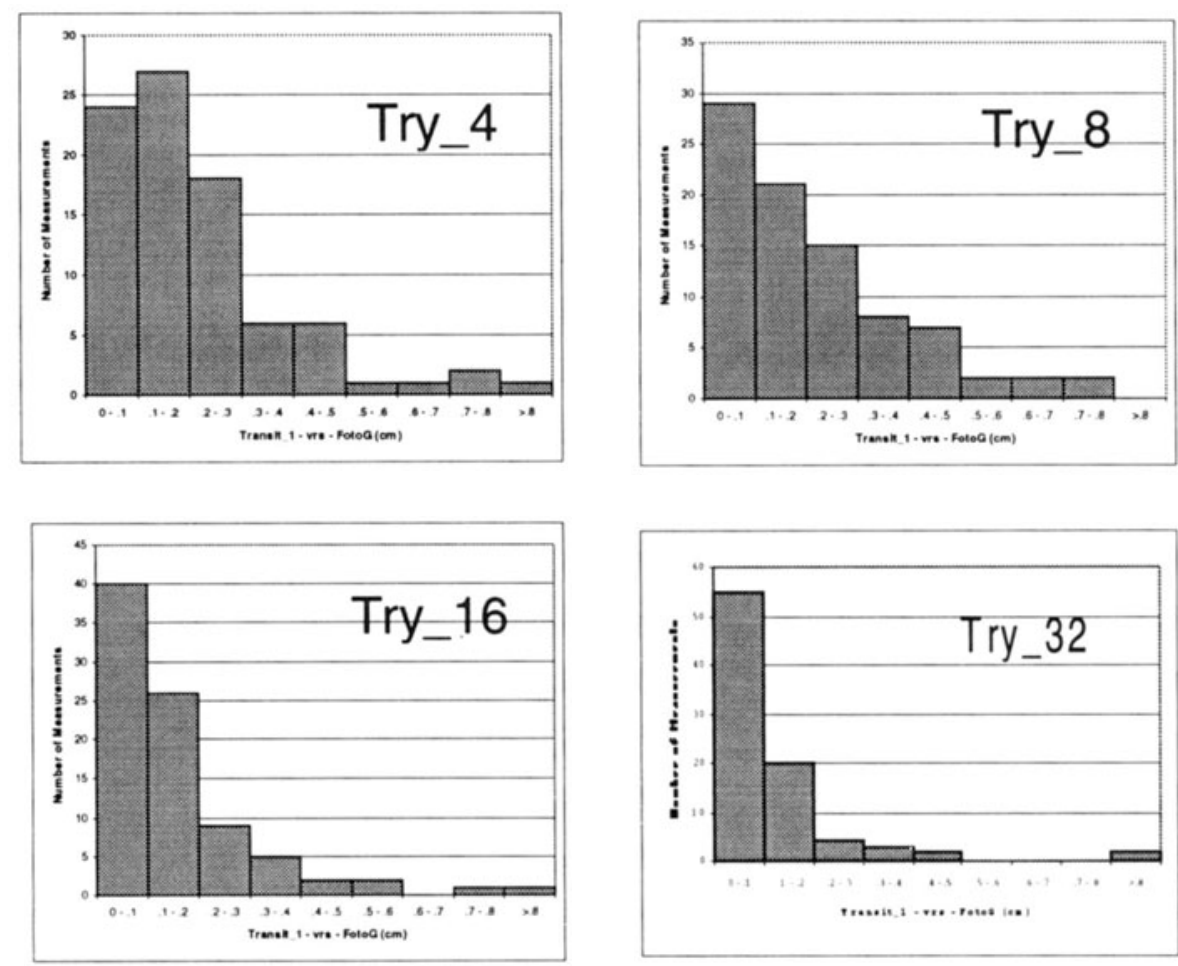

Figure 4 Effect of Number of Control Points on Precision.

The upper two graphs in Figure 5 illustrate the increase in precision from using three images with good geometry as compared to two. However, as illustrated by the lower left graph, adding a fourth image with not quite as strong geometry had a negative effect on the precision. The analyses pictured in the two lower graphs in Figure 5 both employed the use of four images, but the analysis pictured on the left used images with stronger geometry than that on the right.

\section{CONCLUSION}

Photogrammetry, radiography, and reverse engineering reconstruction techniques are effective tools in the non-destructive evaluation of the product life cycle of an assembly. These techniques can be used to forestall problems before they become catastrophic. Research and development continue to improve processes and software development in this area. 

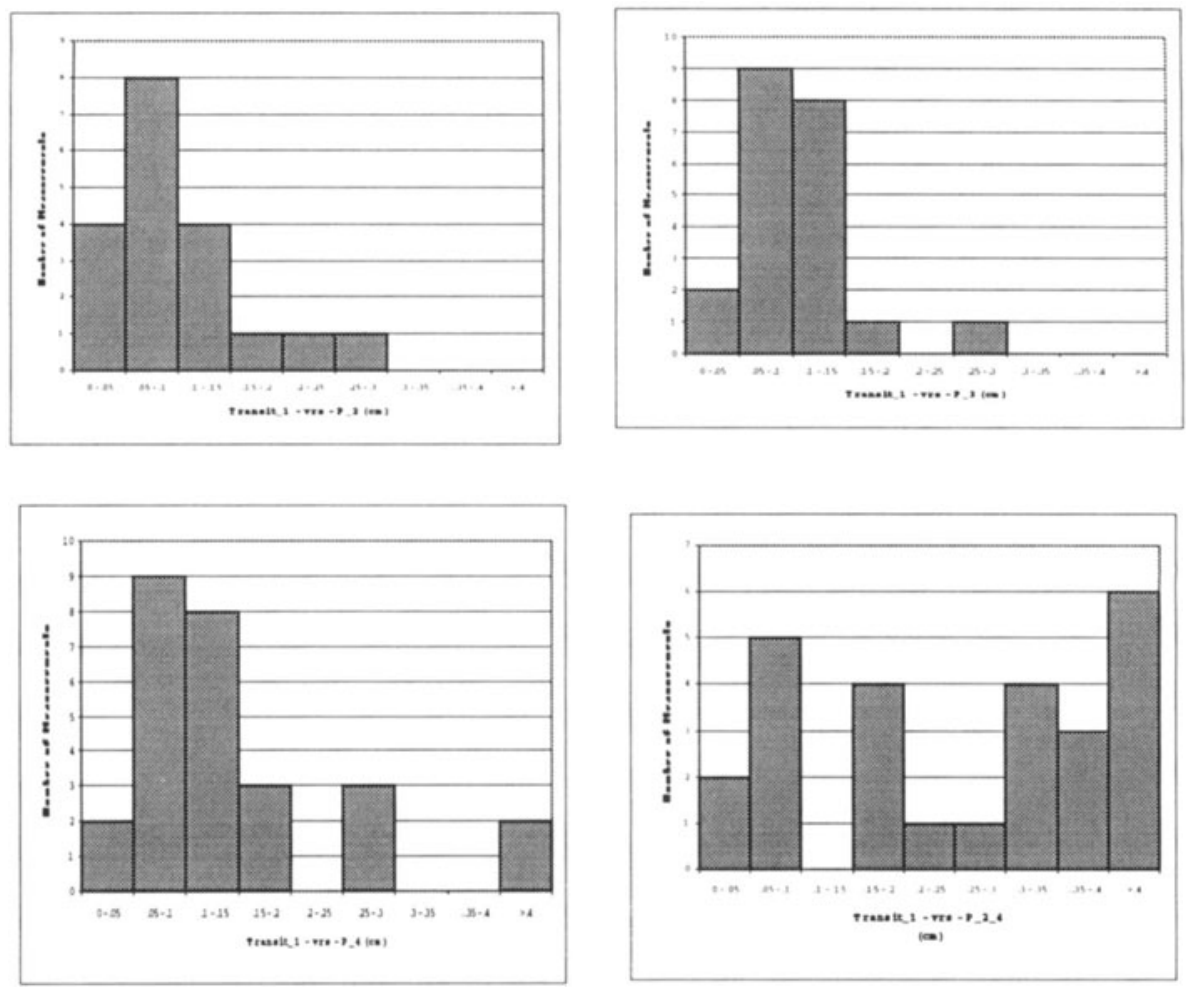

Figure 5 Effect of Number of Images on Precision.

\section{REFERENCES}

[1] Dolin R. M., Hefele J. (1997). As-Built Engineering: A Philosophy for Representing Product Definition, LA-UR-97-3631 Proceedings Nuclear Explosives Design Physics Conference, Livermore, CA.

[2] Hefele, et. al., (1997). Product Characterisation Using Inspection Data in an As-Built Engineering Environment, Proceedings Second Biennial Tri-Laboratory Engineering Conference on Modelling and Simulation, Los Alamos, NM.

[3] Hefele, J. Dolin R. M. (1998). Density Effect of Inspection Data in As-Built Modelling of Parts, Proceedings $2^{\text {nd }}$ International Conference on Engineering Design and Automation, Maui, HI.

[4] Kelley T. A., et. al. (1998). Reverse Engineering Using Computed Tomography, Proceedings $25^{\text {th }}$ Annual Review of Progress in Quantitative Nondestructive Evaluation, Snowbird, UT

[5] Close Range Photogrammetry - Learn About Photogrammetry, http://www.vexcel.com/fotog/learn.html 